\title{
EL TEMPLO DE SAN BIAGIO DE ANTONIO DA SANGALLO EL VIEJO EN MONTEPULCIANO. VERIFICACIÓN DE LA CONSISTENCIA ESTÁTICA PARA SU CONSOLIDACIÓN
}

El templo de San Biagio de Antonio da Sangallo el Viejo. Fuente: Archivo del autor

** Traducción Carlos Humberto Gómez Arciniegas. Arquitecto Universidad Santo Tomás Bucaramanga - MSc. Lancaster University - Lancaster (Inglaterra) - PhD - Politecnico di Milano - Italia

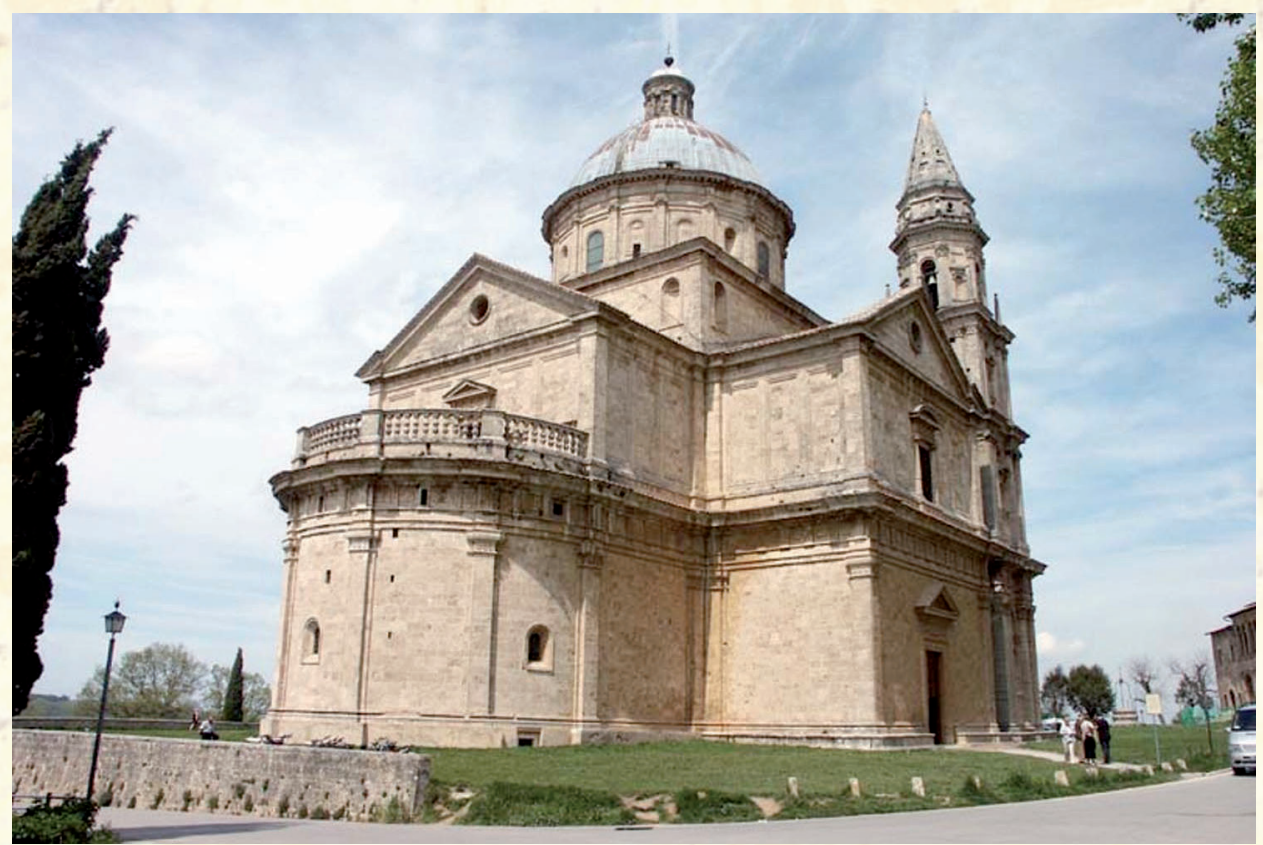

\section{RESUMEN}

En la primavera de 2004, la Superintendencia del Patrimonio Arquitectónico y del Paisaje de las provincias de Siena y Grosseto, aprovechado el andamiaje montado para la restauración que se llevaba a cabo en las fachadas del templo, detectó un extenso patrón de grietas en el recubrimiento externo en mármol travertino, y lo relacionó con análogas "señales" de deterioro presentes en el interior del templo. De particular preocupación son las evidentes manifestaciones de un incipiente aplastamiento en los cuatro pilares esquineros del interior, que de hecho sostienen el sistema cúpula-tambor superpuesto. Se decidió, entonces, promover una campaña de investigación diagnóstica in situ, "no destructiva en lo posible", sobre dichos pilares, además, se concordó sobre la necesidad de proceder a la verificación de otros elementos como la revisión del levantamiento geométrico y arquitectónico existente, el levantamiento del patrón de grietas, la identificación del sistema constructivo de los cuatro pilares, a través de la restitución gráfica de secciones estratigráficas horizontales y análisis numéricos y gráficos para determinar la consistencia estática del sistema portante principal, sin excluir, en relación con el nivel de riesgo que se encuentran, las primeras indicaciones sobre las soluciones para la consolidación.

Por tal motivo se contrató la firma Ser.Tec. S.A.S. Florencia, del Ing. Leonardo Paolini, y el Departamento de Construcciones de la Universidad de Florencia. Los resultados del estudio investigativo han dado lugar al proyecto y obra de la consolidación de los dos primeros pilares (Profesor Giorgio Croci, Roma). En la actualidad se encuentra en curso un programa para determinar la geometría y la técnica constructiva de la cúpula primordialmente. De todo esto se presenta el siguiente informe.

\section{PALABRAS CLAVE}

Arcos, análisis, consolidación, conservación, cargas, concreto, cúpula, fachada, grietas, investigación, pilastras, Renacimiento, tambor, templo, travertino. 


\section{THE CHURCH OF SAN BIAGIO BY ANTONIO DA SANGALLO THE ELDER AT MONTEPULCIANO. STATIC CONSISTENCY CHECKING FOR CONSOLIDATION}

Michele Paradiso*

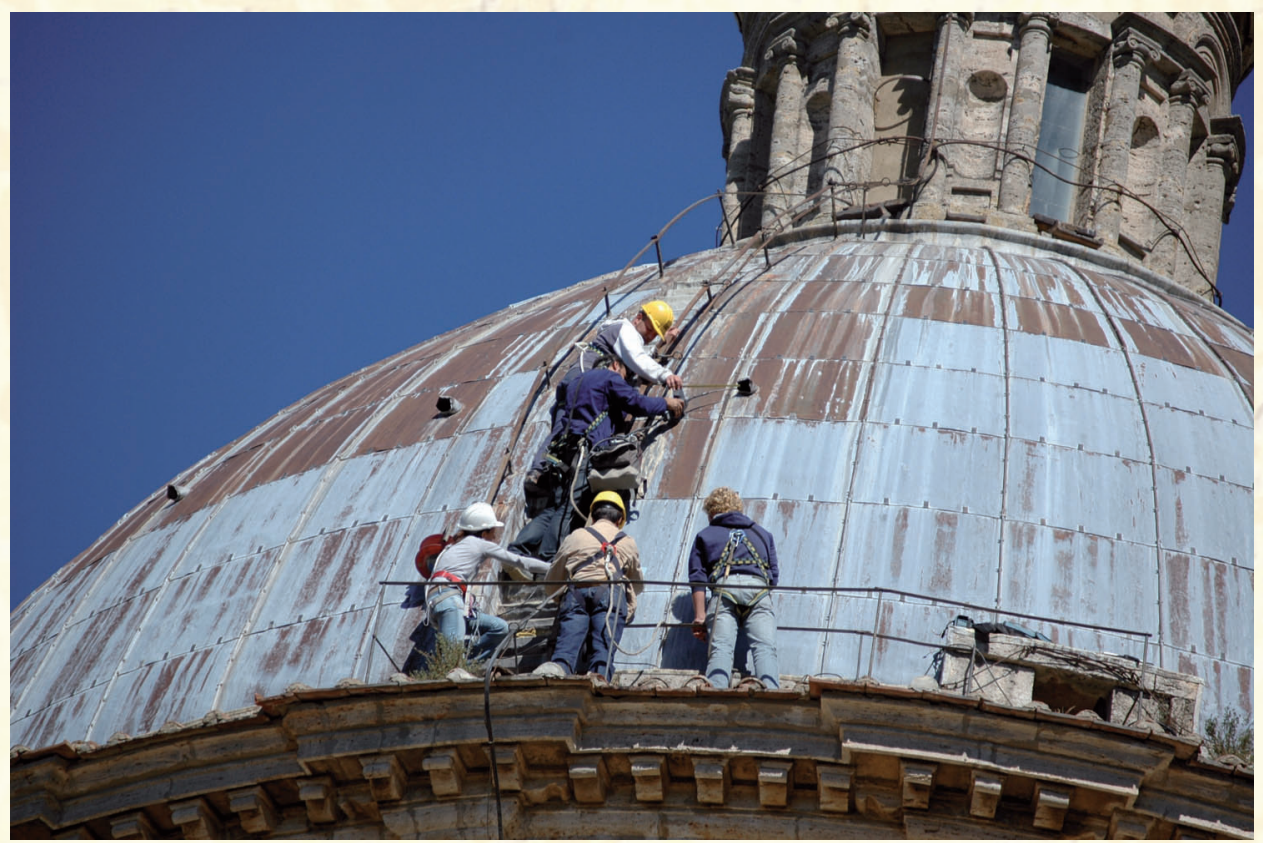

\section{ABSTRACT}

In the spring of 2004, the Superintendency of the Architectural Heritage and Landscape in the provinces of Siena and Grosseto, mounted the scaffolding used for the restoration that took place on the walls of the temple, found an extensive pattern of cracks in the outer coating travertine marble, and related it to similar "signs" of impairment are present inside the temple. Of particular concern are the obvious manifestations of an incipient crushing in four cornerns of the interior pillar, wich in fact support the dome-drum system overlay. It was decided, therefore to promote a campaign of diagnostic investigation in situ, "nondestructive as possible" on these pillars, in addition, agreed on the need for verification of other elements such as the revision of existing architectural and geometric lifting, lifting the cracking pattern, the identification of the construction system of the four pillars, through graphic restitution horizontal stratigraphic section numerical and graphical analysis to determine the consistency static main carrier system, not excluding, in relation to level of risk found, the first indications of the solutions for consolidation.

For this reason, the firm hired Ser.Tec. S.A.S. Florence, Leonardo Paolini Engineering and Construction Department of the University of Florence. The results of the research study have led to the design and construction from the consolidation of the first two pillars (Professor Giorgio Croci, Rome). Currently underway is a program to determine the geometry and construction technique primarily dome. From all this we present the following report.

\section{KEY WORDS}

Arcos, analysis, consolidation, conservation, charges, concrete, dome, facade, cracks, research, pilasters, Renaissance, drum, travertine, temple. 


\section{ABSTRACT (ITALIANO)}

Nella primavera del 2004, la Soprintendenza per i Beni Architettonici e per il Paesaggio per le Province di Siena e Grosseto, approfittando dei ponteggi per il restauro in corso delle facciate del Tempio, prende coscienza di un diffuso quadro fessurativo del rivestimento esterno in travertino e lo mette in relazione con analoghi "segni" di degrado presenti all'interno del Tempio. In particolare preoccupano evidenti fenomeni di schiacciamento incipiente sui quattro pilastri angolari interni, che di fatto sostengono il sistema cupolatamburo soprastante.

Decide così di promuovere una campagna di indagini diagnostiche in situ, "possibilmente non distruttive", su detti pilastri, concordando però sulla necessità, con l'occasione, di procedere alla verifica di alcuni altri elementi, quali il controllo del rilievo geometrico e architettonico esistente, il rilievo del quadro fessurativo, la determinazione della tecnologia costruttiva dei quattro pilastri, attraverso la restituzione di sezioni stratigrafiche orizzontali, nonché analisi numeriche e grafiche atte a determinare la consistenza statica del sistema portante principale, senza escludere, in relazione al livello di rischio accertato, prime indicazioni sulle soluzioni per il consolidamento. Vengono così coinvolti lo studio Ser.Tec. s.a.s. di Firenze, dell'ing. Leonardo Paolini, e il Dipartimento di Costruzioni dell'Università degli Studi di Firenze. I risultati dell'indagine hanno poi portato alla progettazione e realizzazione del consolidamento dei primi due pilastri (Prof. Giorgio Croci, Roma). Attualmente é in corso una campagna atta ad accertare principalmente la geometria e la tecnica costruttiva della cupola. Di tutto ciò si da conto di seguito.

\section{PAROLE CHIAVE:}

Arcos, l'analisi, il consolidamento, lo stoccaggio, carico, cemento, cupola, facciata, crepe, la ricerca, lesene, Rinascita, tamburi, tempio, travertino.

\section{PREFACIO}

El profesor Michele Paradiso, docente e investigador de la Universidad de los Estudios de Florencia (Italia) evidencia con este artículo, escrito para la Revista M, la importancia de la gran labor investigativa que encierra el campo de la restauración de monumentos arquitectónicos. De su gran copiosa experiencia académica y profesional, el autor escoge en esta ocasión un compendio de las experiencias y apreciaciones recopiladas a través de las obras de consolidación de la estructura del templo de San Biagio, construido por Antonio da Sangallo el Viejo. El santuario yace en fondo a una vía bordeada de cipreses en inmediaciones de la ciudad de Montepulciano, conocido asentamiento de la Provincia de Siena (Región Toscana) por la riqueza de su patrimonio territorial.' Este edificio religioso compendia claramente la suma de los estudios del Renacimiento sobre la planta de cruz griega, retomada y materializada en Italia central durante esta época por arquitectos como Filippo Brunelleschi, Donato de Bramante y Miguel Ángel Buonarroti. Antonio da Sangallo no fue la excepción, al inspirarse en la basílica de Santa María delle Carceri en Prato (Toscana), diseñada una generación antes por su hermano Giuliano. ${ }^{2}$ Sin embargo, el tiempo, al hacer caso omiso de la belleza de este templo, ha marcado una serie de lesiones en su estructura, las cuales han concentrado a un grupo de técnicos, estudiosos e investigadores, preocupados por la salvaguarda y recuperación del patrimonio arquitectónico italiano, alrededor del caso de la iglesia de San Biagio, llamado también templo por su monumentalidad. En consecuencia, el profesor Paradiso incluye un registro fotográfico donde se

http://www.cretedisiena.com/paesi/Montepulciano/sbiagio.htm

2 http://www.icvbc.cnr.it/bivi/schede/Toscana/montepulciano//biagio.htm 
muestra el estado actual del templo que, a pesar de los problemas que se ilustran en el cuerpo del artículo, en ningún momento le restan imponencia a elementos como la cúpula central o su fachada principal, esta última con un esquema de composición repetitivo pero con algunas peculiaridades constructivas, claramente señaladas por el autor, como lo es la referencia hecha al momento de hablar del proyecto original que preveía la construcción de dos torres simétricas, una de ellas nunca terminada. Asimismo, el artículo hace énfasis en las características del sistema constructivo y estructural, el allí omnipresente mármol travertino y muchos otros elementos ornamentales como columnas embebidas, lesenas y hasta peculiaridades de la cúpula que no hacen otra cosa que justificar plenamente los trabajos de consolidación para conservar esta joya del patrimonio arquitectónico, heredado al mundo por Italia y que, junto a otras más, han dado vida a un verdadero catálogo de la estética del Renacimiento. ${ }^{3}$

$$
\begin{array}{r}
\text { Carlos Humberto Gómez Arciniegas } \\
\text { Arquitecto - Universidad Santo Tomás - Bucaramanga (Colombia) } \\
\text { MSc - Lancaster University - Lancaster (Inglaterra) } \\
\text { PHD - Politecnico di Milano - Italia }
\end{array}
$$

\section{ELEMENTOS CARACTERÍSTICOS DE LA ARQUITECTURA DEL TEMPLO}

La ubicación aislada del edificio, en las afueras del casco urbano, permitió a Antonio da Sangallo desarrollar libremente la idea de un edificio de planta central. Se trata de un cuerpo de cruz griega. En la parte sur se yuxtapone una tribuna absidal, de medio punto y coronada por una balaustrada, de donde se recaba la sacristía, mientras que, sobre el extremo opuesto de la cruz, que debe ser considerado como la fachada principal de la iglesia, se encuentran dos torres (campaniles) que se insertan entre los brazos de la cruz y bordean el volumen pero sin fusionarse al cuerpo del edificio (Ver Fig. I).

El uso de mármol travertino proveniente de S. Albino, patinado por el paso del tiempo y con sus reflejos unas veces color rosa, y otras veces dorados, proporciona una singular uniformidad material y cromática. El edificio se yergue sobre un amplio basamento realzado, interrumpido solamente por las tres puertas ubicadas en la parte central de cada frente. Exteriormente, la fachada se compone de dos pisos coronados por un amplio frontón triangular, cuya linealidad se ve interrumpida por un óculo que se abre en el centro. El portal está coronado por un simple frontón triangular mientras que en los costados cada pared está delimitada por sólidas lesenas o bandas decorativas dóricas.

El ábside semicircular que prolonga el brazo posterior de la cruz griega, no es visible al interior, ya que la pared en la que se apoya el altar mayor, divide esta zona en pro del perfecto desarrollo de los cuatro brazos.

El esquema externo se repite en el interior, donde la cruz griega es exactamente respetada. De igual manera, las columnas y pilastras dóricas se levantan sobre el basamento que corre a lo largo de todo el perímetro interior sigue, a su vez, el mismo recorrido del entablamento y de la cornisa, muy marcada ésta última aquí, también. Por encima de la cornisa se emplazan los arcos de las bóvedas que cubren cada brazo de la cruz. Por último, en la parte superior del edificio, al centro del cuerpo en forma de cruz y evidenciada por la forma cuadrada del ático, surge la estructura cilíndrica del tambor coronado por la cúpula (Ver Fig. 2).

3 Listri M., Toscana. Vedute, chiese, musei, arte, palazzi, ville, giardini, Rizzoli, 2010 


\section{EL PATRÓN DE GRIETAS}

Los trabajos de levantamiento permitieron establecer y documentar un interesante patrón de grietas. En las fachadas externas el recubrimiento de mármol travertino aparece deteriorado casi en todas partes, hasta el punto de dar la impresión de una textura y cuerpo en progresivo deterioro. Exfoliaciones, vacíos (lagunas) y eyecciones de material se extienden por doquier, en un proceso lento pero continuo. En algunos casos, las lesiones se acentúan en las juntas entre piedra y piedra, en otros, se alternan a lo largo de su desarrollo longitudinal con uno que otro paliativo, lo que deriva posiciones localizadas de debilidad. De las cuatro fachadas, la este y la oeste denuncian la situación más preocupante, con lesiones verticales en el centro, suficientes para sugerir una tendencia macro cinemática ${ }^{4}$ que aleja el cuerpo norte del cuerpo sur (ver Figura 3)

Este comportamiento se ratifica con la presencia de dos grandes cadenas, situadas a la altura de la línea de impostas de las bóvedas de cañón, de los cuatro brazos, sobre el paramento interior de la fachada. El patrón de grietas interno concuerda con el externo (las principales lesiones se conectan). La cúpula, estucada por dentro y recubierta con láminas de plomo en el exterior, las cuales sustituyeron en 1605 las originales tejas de escama en barro vitrificado, muestra cuatro sutiles "lesiones meridianas" ligadas a los planos cardinales verticales. Los grandes arcos portantes presentan lesiones en su intradós.

\section{LAS LESIONES EN LAS PILASTRAS ESQUINERAS}

Gracias a los andamios armados alrededor de dos de las cuatro pilastras esquineras internas, que sostienen el tambor y la cúpula, fue posible detectar el conjunto de grietas. Las pilastras esquineras analizadas son las que se encuentran dispuestas en la diagonal norteoeste sur-este, línea que intercepta el campanario que no fue terminado. Es así que se evidenciaron aun más los fenómenos de aplastamiento de las columnas de travertino y las expulsiones de material: las que se produjeron, las compensadas y las más preocupantes, en fase de inminente desprendimiento. En algunas zonas, los segmentos superiores de las pilastras resultan lesionados, con una apertura de la lesión de hasta medio centímetro y con una profundidad de hasta quince centímetros (30\% de la profundidad de la totalidad del elemento lapídeo), situación confirmada con el simple golpear de los nudillos o de algún instrumento (Ver Fig. 4).

Fotos del archivo Alinari permiten documentar la presencia de estas lesiones ya a comienzos del siglo $X X$, el fenómeno de aplastamiento es también acompañado por la pérdida de la verticalidad del nivel interior de la columna con una tendencia a "pandearse".

\section{LA TECNOLOGÍA CONSTRUCTIVA}

Se llevaron a cabo amplias pesquisas para identificar el método de construcción de los muros con los cuales se conformó la parte basamental del templo, para apoyar el tambor sobre el que descansa la cúpula central. Cuatro reconocimientos introspectivos específicos y bien encaminados se llevaron a cabo mediante una extracción de núcleo con un diámetro de $80 \mathrm{~mm}$ provenientes de las dos pilastras analizadas. (Ver Fig. 5)

En las muestras obtenidas se llevaron a cabo pruebas instrumentales para verificar las propiedades químicas y físicas de los materiales. Los núcleos estaban constituidos por un

$4 \quad$ Nota del traductor: de cinemática o rama de la mecánica que estudia el movimiento de un cuerpo o un sistema de cuerpos sin consideración dada a su masa o las fuerzas que actúan sobre él. Fuente. /www.rae.es 

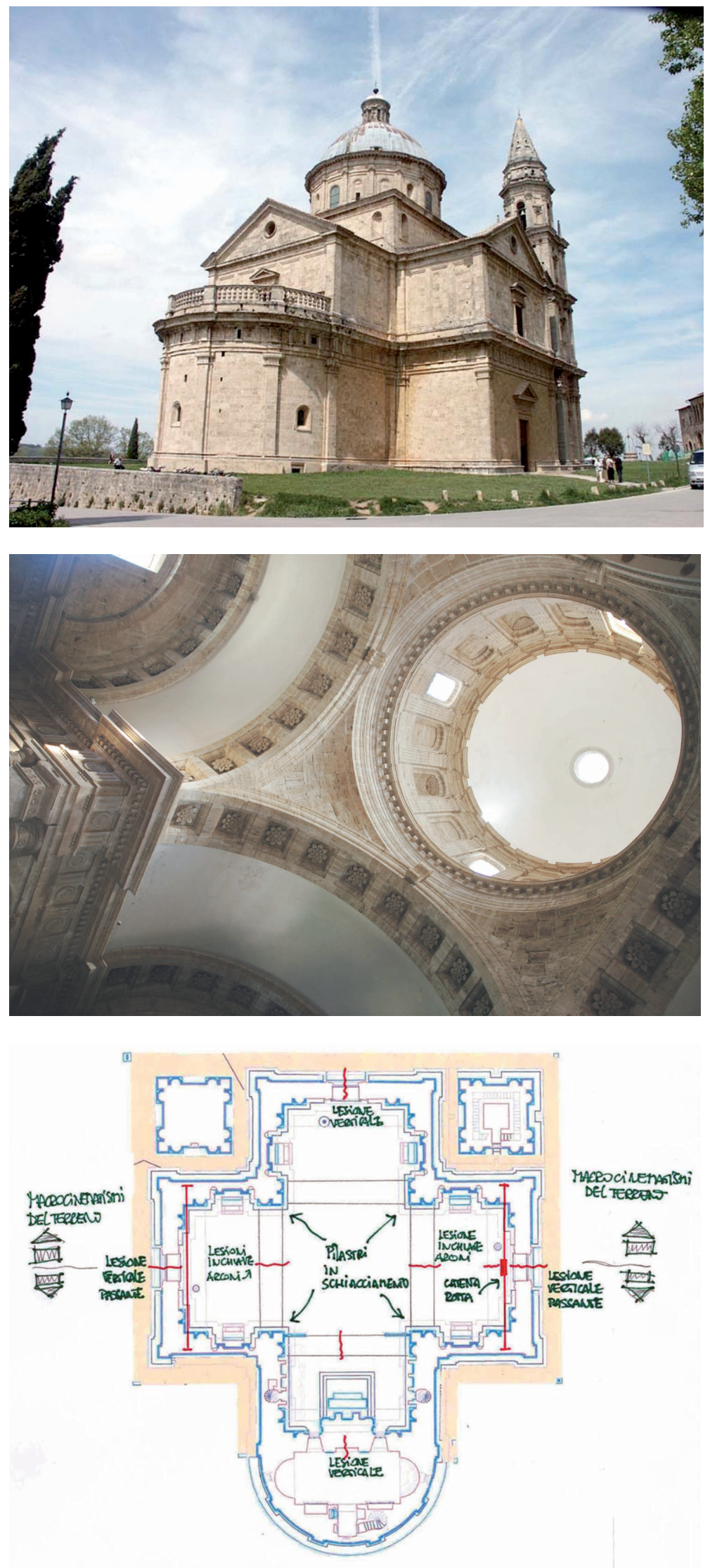

Fig. I. El templo de San Biagio de Antonio da Sangallo el Viejo

Fig. 2. El conjunto cúpula-tambor-arcospilastras

Fig. 3. Hipótesis sobre macro cinematismos en curso 
hormigón cuyo árido (material granulado) iba desde la arenisca hasta el cemento calcáreo muy compacto, desde piedra caliza margosa hasta grano fino, areniscas calcáreas, travertinos, piedra caliza organogénica y ladrillos. Todos estos materiales están unidos por un mortero en general durable, resistente, elaborado con arena local bastante madura y un conglomerante carbonático de aspecto no homogéneo con textura variable. De igual modo, el árido natural tiene una mejor característica física que las del mismo mortero. Por lo tanto, el elemento débil de esta mezcla, que caracteriza la mampostería, resulta ser el mortero que pega el grueso árido del "concreto".

Otra característica estratigráfica, fundamental para la justificación de las causas del patrón de agrietamientos en el monumento, es la forma de los sillares (bloques de mármol) de los paramentos externos en travertino, que presentan un aspecto de pirámide troncada, con la superficie de contacto para emboquillado presente solamente en la superficie externa. Esta situación, particularmente crítica se presenta en las cuatro columnas esquineras que sostienen los arcos que soportan el tambor. De hecho, el elemento construido, implica la formación de tres elementos que sobresalen notablemente del núcleo central: la lesena de borde y las dos columnas laterales de media caña (embebidas) las cuales presentan la mayoría de los agrietamientos detectados (Ver Figura 6). Cabe anotar que aún no se ha determinado completamente cuál es el sistema constructivo de dichas columnas.

\section{EL ANÁLISIS NUMÉRICO}

Se llevó a cabo el análisis numérico sobre el modelo tridimensional del sistema portante cúpula-tambor-pilastras, con métodos tanto pre-modernos con carácter sintético-gráfico como software especializado con hipótesis de materiales de mampostería con un comportamiento no-tensión (SVM, Aedes Software, San Miniato di Pisa), códigos de cálculo "general purpose” para ingeniería civil (Straus7). Se prestó especial atención al comportamiento de las pilastras esquineras, donde el modelado respetó el sistema estructural a muro de relleno, y a las diferencias de comportamiento mecánico entre el paramento en sillares de mármol travertino y la configuración del relleno interno. ${ }^{5}$ El nivel de tensión, verificado al pie de las pilastras esquineras muestra cómo la hipótesis de relleno interno "no cooperante" se relaciona con los agrietamientos, caracterizados por lesiones por aplastamiento que afectan principalmente las columnas embebidas. Es probable que aún este fenómeno no se haya extendido por todo el relleno aunque la situación real puede alcanzar un nivel intermedio. Seguramente el fenómeno progresa lenta pero irreversiblemente.

\section{LA CONSOLIDACIÓN DE LAS PILASTRAS ESQUINERAS}

La situación de inestabilidad detectada y confirmada así como el historial estático del monumento, caracterizado por una continua serie de reparaciones de emergencia, obligaría a la creación de un fondo para detectar la solución técnica definitiva para resolver la problemática estructural actual, una vez que se sepa más sobre la consistencia del relleno, monitoreando los cuatro pilares en toda su altura con técnicas no destructivas así como la naturaleza y consistencia de los cimientos y el comportamiento del terreno rocoso sobre el cual se apoya completamente el Templo de San Biagio.

Una primera hipótesis contempló la posibilidad de sustituir el relleno de las monumentales pilastras por un entramado continuo en travertino, a través de una desarticulación iniciada desde el ápice del paramento con la ayuda de una estructura metálica que delimite y con-

$5 \quad$ Nota aclaratoria del traductor: se hace referencia a un sistema constructivo denominado Emplectum por los romanos pero que según Vitrubio y Plinio era de origen griego, análogo al opus caementicium. De cualquier modo, el sistema consistía en crear dos hojas exteriores de sillares de piedra, rellenas de un mortero de cal con arena y cascotes. Caviasca Massimiliano, L'evoluzione della tipologia e la materia costruttiva. II muro a emplecton, Politecnico di Milano. Matua, 2008 

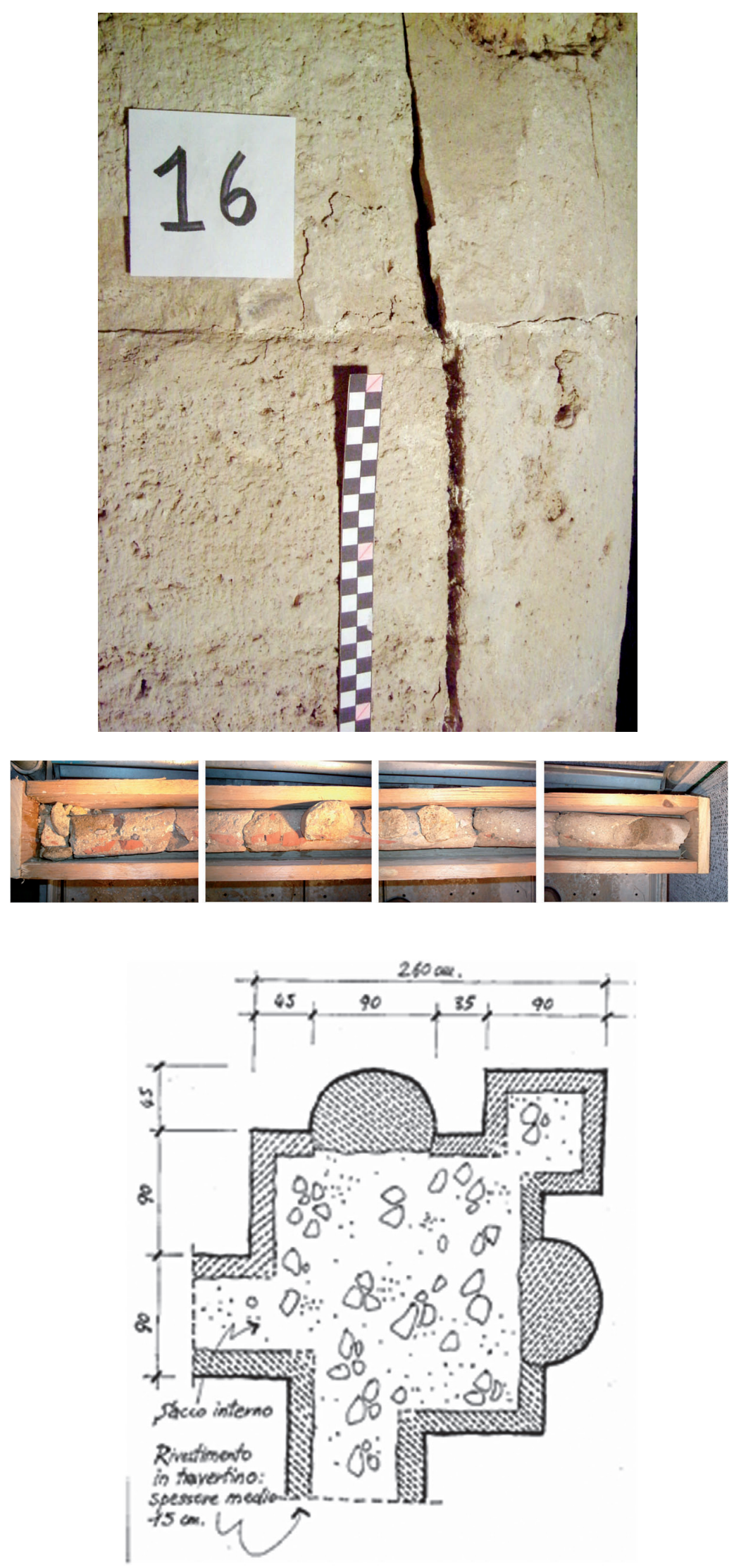

Fig. 4. Lesión por aplastamiento en un pilar esquinero

Fig. 5. Extracción de núcleo (carotaje) efectuada a 2.70 metros de altura de la base

Fig. 6. Geometría básica de las cuatro pilastras esquineras que soportan el tambor de la cúpula 
tenga. La parte principal de la intervención habría sido la construcción de un sistema de apuntalamiento, constituido por un entramado reticular que sostuviese las cargas verticales del sistema cúpula-tambor, que descargue de este modo los arcos y pilastras subyacentes y permita, por lo tanto, el "remiendo", la regeneración y sostén de los elementos que conforman los muros de las pilastras.

Las incógnitas sobre la naturaleza y la consistencia del terreno y del piso de la nave, sobre el cual se habrían tenido que apoyar las torres reticulares, además del considerable compromiso financiero necesario, llevaron a adoptar otras soluciones. La consolidación en curso de los pilares es el "rededor" -condicionado por la cercanía de las pilastras a los muros perimetrales de los cuatro brazos- hecho con cables de FRP, adecuadamente tensados, repartidos generosamente a lo largo de toda la altura de las pilastras, con el fin de aumentar el umbral de crisis por aplastamiento. Esta intervención, que llena todo el volumen de las pilastras bajo el mismo criterio, confrontado con la tecnología actual de los "tensores anti-expulsivos", se debe considerar, una vez más, como una intervención de emergencia.

\section{LA ESTRUCTURA DE LA CÚPULA DEL TEMPLO}

La cúpula de San Biagio fue construida entre I 536 y I544, aproximadamente, año en el que se hace un pago para el revestimiento de la cúpula. Esta es una de las cúpulas del Renacimiento más realzadas, lo cual no sólo permite reducir los empujes horizontales sino también evitar que ésta parezca estar muy hundida al observarla desde abajo. Hasta la fecha hay incertidumbre tanto por los elementos geométricos de los dos sextos (interno y externo) de los relativos centros de curvatura y de la consistencia del casquete Algunos autores (Geymüller, Barchi) proponen en su representación gráfica un doble cuerpo (Ver Figura 7), otros, recientemente, (Giorgi, Fagnani, Meloni y otros) una cúpula a sección llena (Ver Figura 8).

Recientes indagaciones con video inspección en las tomas de aireación en el trasdós de la cúpula, complementados con datos y comprobaciones efectuadas durante los años anteriores han demostrado en forma concluyente la presencia de un vacío, sin embargo, se supone además que este vacío se interrumpe en correspondencia con los planos cardenales verticales, para formar así cuatro costados llenos, cuya colocación se proyecta sobre las claves de los grandes arcos inferiores de descarga. La investigación se llevó a cabo por técnicos de la SMG srl Ingeniería Experimental, de Perugia, en nombre de la Superintendencia, coordinado por el Profesor Lorenzo Capineri del Departamento de Electrónica y Telecomunicaciones de la Universidad de Florencia (Ver Figura 9).

\section{CONCLUSIONES}

La posibilidad ofrecida a los autores de la Superintendencia de Patrimonio Arquitectónico y de Paisaje de las provincias de Siena y Grosseto, para evaluar, a través de una serie de investigaciones la consistencia estática del Templo de San Biagio di Antonio da Sangallo el Viejo, llevó a la conclusión de que el monumento realmente vive una situación crítica, develada por una serie de notorios agrietamientos que registran una situación particular en las pilastras esquineras en su momento más preocupante. Esta situación pertenece a la historia de la estática del Templo, es documentable y documentada, especialmente en lo que respecta a un período más o menos reciente.

La medida en que esta situación crítica haya sido provocada por algunas decisiones de diseño o de tecnología constructiva, sólo se podría llegar a saber cuando se haya finalizado el análisis y el estudio de la gran cantidad de documentos históricos, relativos a la cons- 

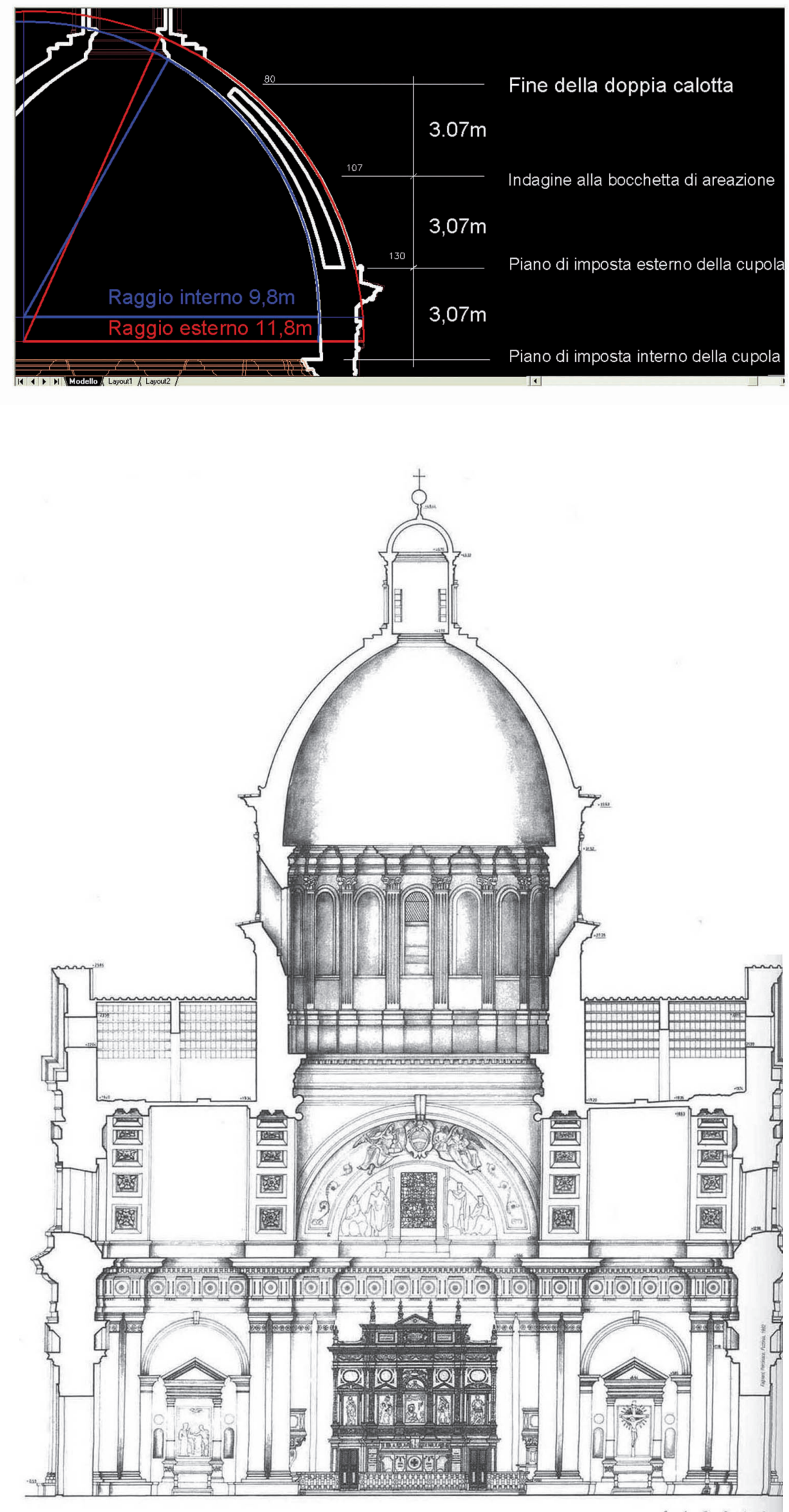

Fig. 7 La estructura a doble casquete

Fig. 8 La sección transversal del templo según el levantamiento de Meloni 
Fig. 9 Video-endoscopia practicada en la cubierta exterior

Fuente: Archivo del autor

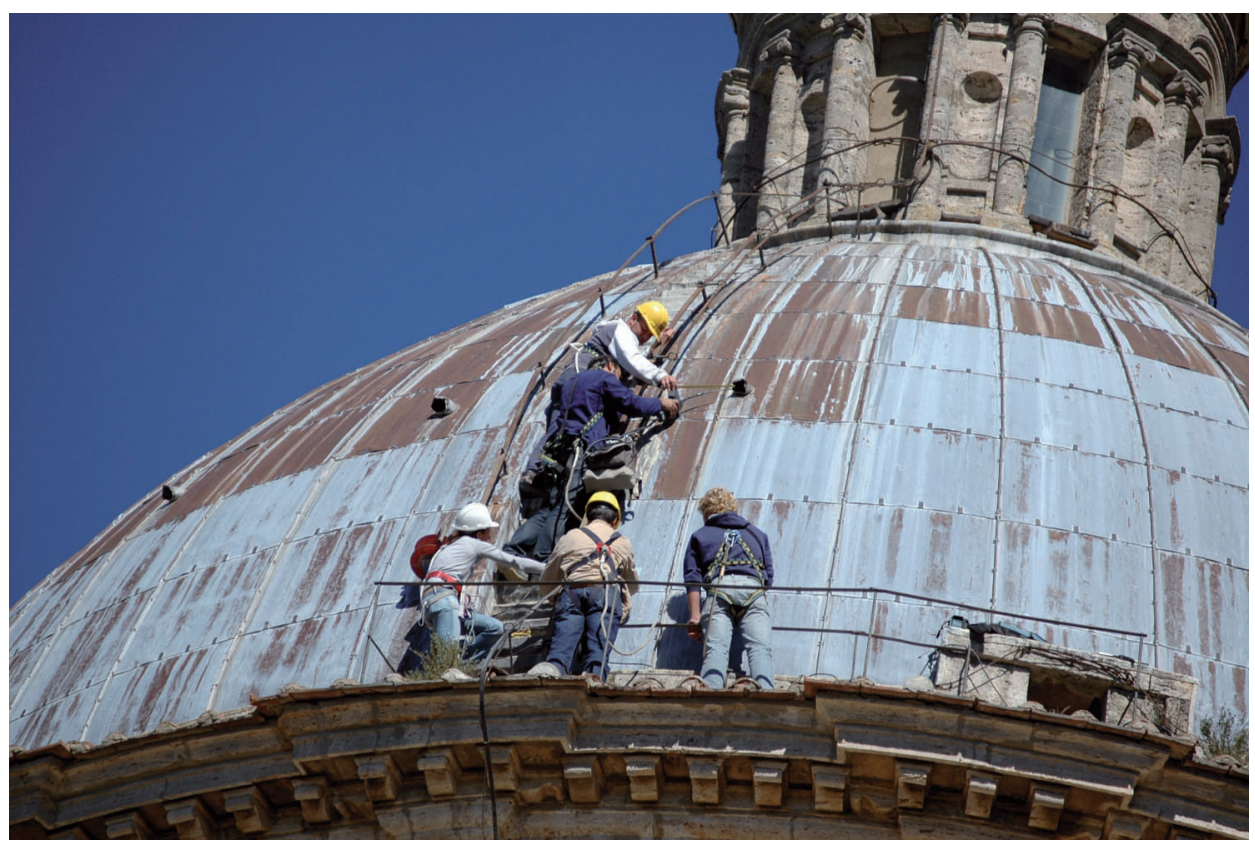

trucción del templo, que aún se encuentran sin catalogar en el archivo histórico municipal de Montepulciano.

También podrían ser útiles una serie de investigaciones con tecnología GeoRadar que se espera poder ejecutar sobre el piso de la Iglesia y en el intradós de la cúpula. Subsiste, sin embargo, la necesidad ineludible de interrumpir la cadena de intervenciones de emergencia, de soluciones paliativas, adoptadas hasta hace poco, para encontrar una solución técnica que resuelva de manera definitiva la situación de este elemento, tal vez considerado como la más prestigiosa arquitectura posterior a Brunelleschi.

\section{BIBLIOGRAFÍA}

COZZI M., "Antonio da Sangallo il Vecchio e l'architettura del Cinquecento in Valdichiana", Sagep Editrice, Genova, 1992

GIORGI L., "Antonio da Sangallo il Vecchio e Andrea Pozzo a Montepulciano: il Tempio della Madonna di San Biagio e la Chiesa del Gesù", Le Balze, Montepulciano, 1999

SATZINGER G., "Antonio da Sangallo der Altere und die Madonna di San Biagio bei Montepulciano", Erns Wasmuth Verlag, Tubingen, 1991

STEGMANN C., GEYMULLER H., "Die Architektur der Renaissance in Toscana“", Munchen, 1885-1909

VASARI G., "Le Vite dei più eccellenti pittori scultori e architettori...,", vita di Giuliano e Antonio da Sangallo ), a cura di Rossana Bettarini, commento secolare a cura di Paola Barocchi, SPES, Firenze 1976, vol.IV, pp. I3I-I32

MELONI J, “La Madonna di San Biagio - Montepulciano - dal rilievo al disegno”, tesi di laurea, relatore Prof. Maria Luisa Bartoli. a.a. 2003-2004, Facoltà di Architettura, Università degli Studi di Firenze. 
MEZZASOMA F., SERPA F., "IL tempio di san Biagio in Montepulciano di Antonio da Sangallo il Vecchio:analisi sulla consistenza statica e ipotesi di consolidamento", tesi di laurea, relatore Prof. Michele Paradiso, aa. 2004-2005, Facolta' di Architettura, Univerità degli Studi di Firenze.

"Mappature interventi colonne centrali Tempio di san Biagio", Mannelli Costruzioni Firenze, Studio Croci Roma, Report Interno. 